\title{
Endoscopic medial maxillectomy combines outside-in draf 3 approach for sinonasal squamous cell carcinoma
}

\author{
Tengchin Wang* \\ Department of otolaryngology, Tainan municipal hospital, Tainan City, Taiwan
}

\begin{abstract}
For treatment of sinonasal inverted papilloma (IP) or malignant disease, it is essential to have a definite diagnosis, and to select the appropriate surgical approach. Endoscopic medial maxillectomy and outside-in Draf 3 approach are good choice as in maxillary and fronatal disease. We successfully treated sinonasal IP with squamous cell carcinoma transformation in a 46-year-old male patient without navigation system guided.
\end{abstract}

\section{Introduction}

Surgical resection is the main choice for managing sinonasal malignant tumors. However, accessing to the lesion inside the frontal sinus is quite challenging to physicians, particularly in situations lacking navigation system guidance; moreover, locating the frontal recess becomes extremely difficult under the circumstances of tumor infiltration. Several techniques have been developed for overcoming the challenges. An outside-in Draft 3 technique enables timely delineating safe dissection margin and preventing penetrating through frontal recess in the beginning [1]. This is a reliable technique for resecting sinonasal tumors, particularly in the frontal area.

\section{Case report}

We report a 46-year-old male who suffered from right epistaxis for several months. He received examination in our department, a papillomatous lesion was identified in right nasal cavity (Figure 1). Biopsy reports inverted papilloma. Computed tomography with contrast displays an enhanced tumor involving right ethmoid, orifice of maxillary sinus (Figure 2a), and there is bony decay of nasal lacrimal duct (NLD) (Figure 2b). The frontal recess is also invaded by tumor that induces secondary right frontal sinusitis (Figure 2c). Concerning potential malignant transformation of inverted papilloma, we arranged eradicated approaches for the patient. After debuking the tumor, the origination at lateral nasal wall was identified. Therefore, we performed endoscopic medial maxillectomy with ethmoidectomy. The superior boundary of resection is fovea ethmodalis (Figure 3a), then incision is carried down to nasal floor and backward till sphenoid rostrum (Figure 3c). The main tumor, medial maxillary wall, and inferior turbinate are removed in one piece, the remaining antral mucosa is relative healthy without tumor seeding (Figure $3 \mathrm{~b}$ ). For possibly residual tumor in the frontal recess, we perform outside- in Draf 3 approach to avoid penetrating through tumor that may cause further contamination into frontal sinus. We delineated frontal sinus floor early and directly dissected into frontal sinus, going beyond the tumor, then backing to eradicate frontal recess lesion eventually (Figure $3 \mathrm{~d}$ ). There is no bony invasion of frontal recess and just a mild secondary frontal sinusitis. At the end, dacryocystorhinostomy was performed to restore the lacrimal draining system.

No sequelas was left after surgery. The patient discharged 3 days later smoothly. Pathology displays inverted papilloma with invasive non-keratinized squamous cell carcinoma, moderately differentiated. Focal lymphovascular invasion is noted, adjuvant radiotherapy was scheduled. Lacrimal stent was removed 12 weeks after operation, the patient doesn't complain epiphora. We have followed him for 22 months without seeing any recurrence (Figure 4).

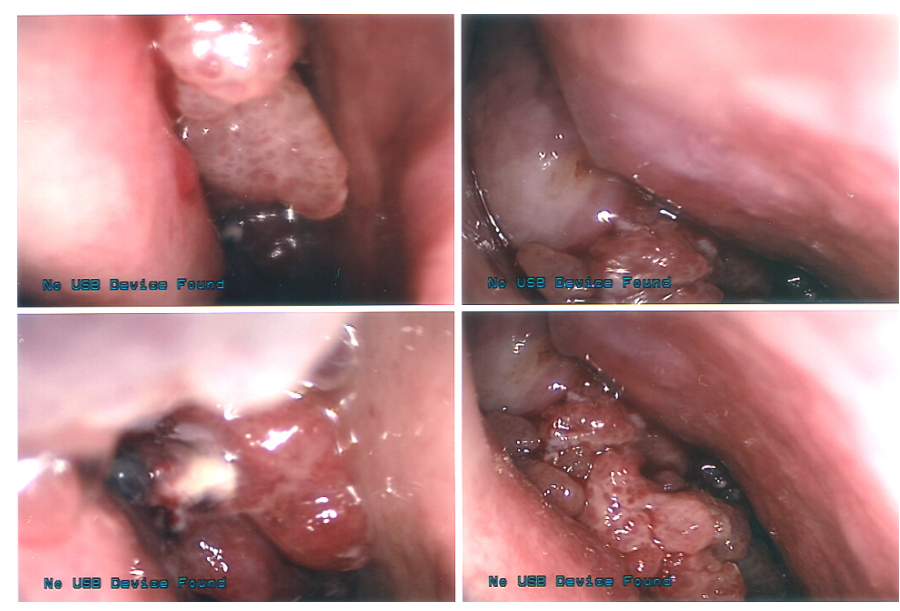

Figure 1. A papillomatous lesion obstructs right nasal cavity with superficial necrosis Middle turbinate and axilla region can't be identified.

Correspondence to: Tengchin Wang, Department of otolaryngology, Tainan municipal hospital No.670, Chongde Rd., East Dist., Tainan City 701, Taiwan, Tel: +886-6-2609926; E-mail: tengchin27@hotmail.com

Key words: endoscopic medial maxillectomy, outside-in Draf 3 approach

Received: October 20, 2017; Accepted: November 23, 2017; Published: November 27, 2017 

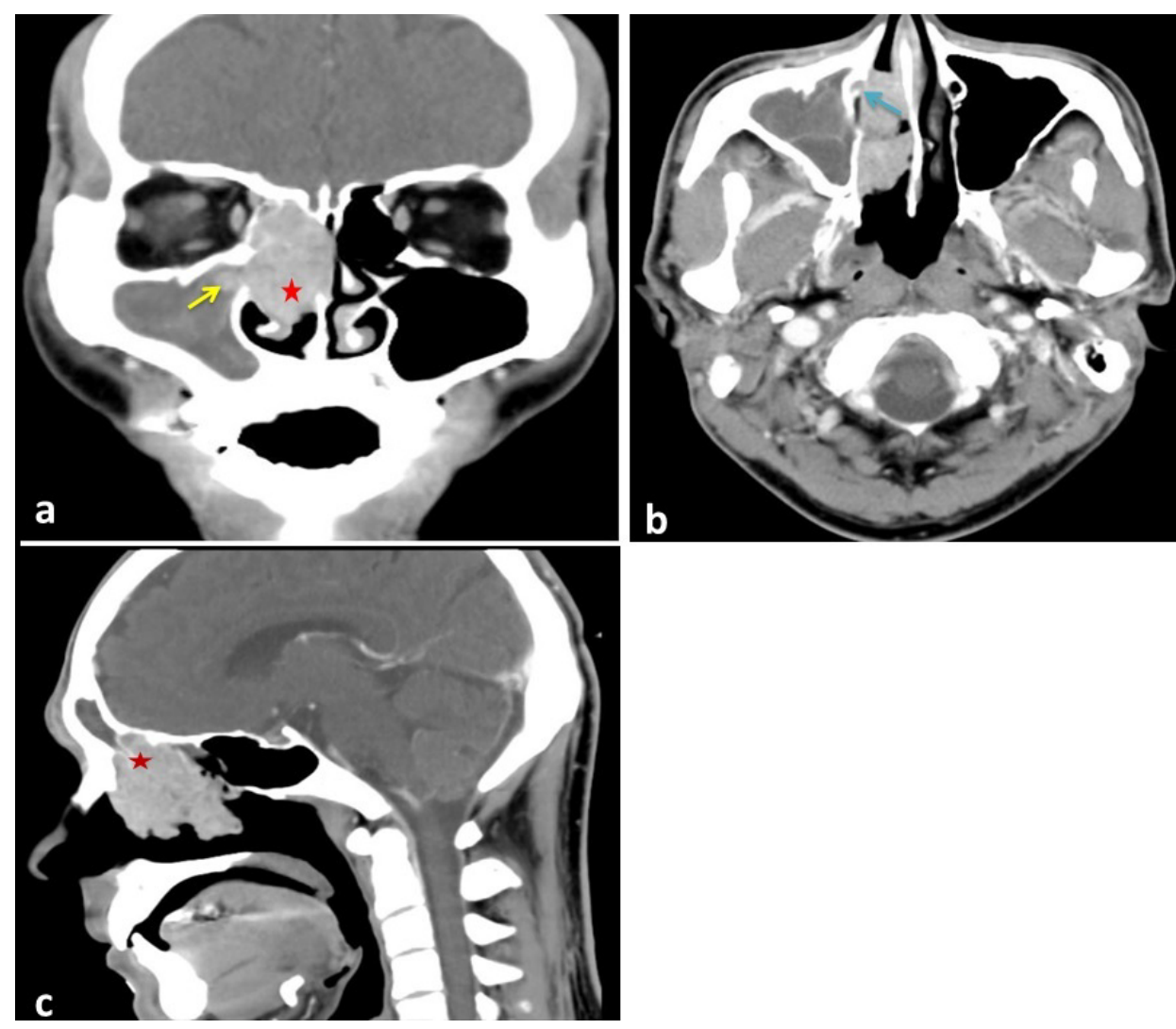

Figure 2. (a) red asterisk indicates the mass mainly involves ethmoid sinus, intra-maxillary extension is also visible (yello arrow); (b) bony decay of lacrimal bone with soft tissue infiltrates into nasolacrimal duct (blue arrow); (c) tumor invades frontal recess and obstructs draining pathway of frontal sinus.
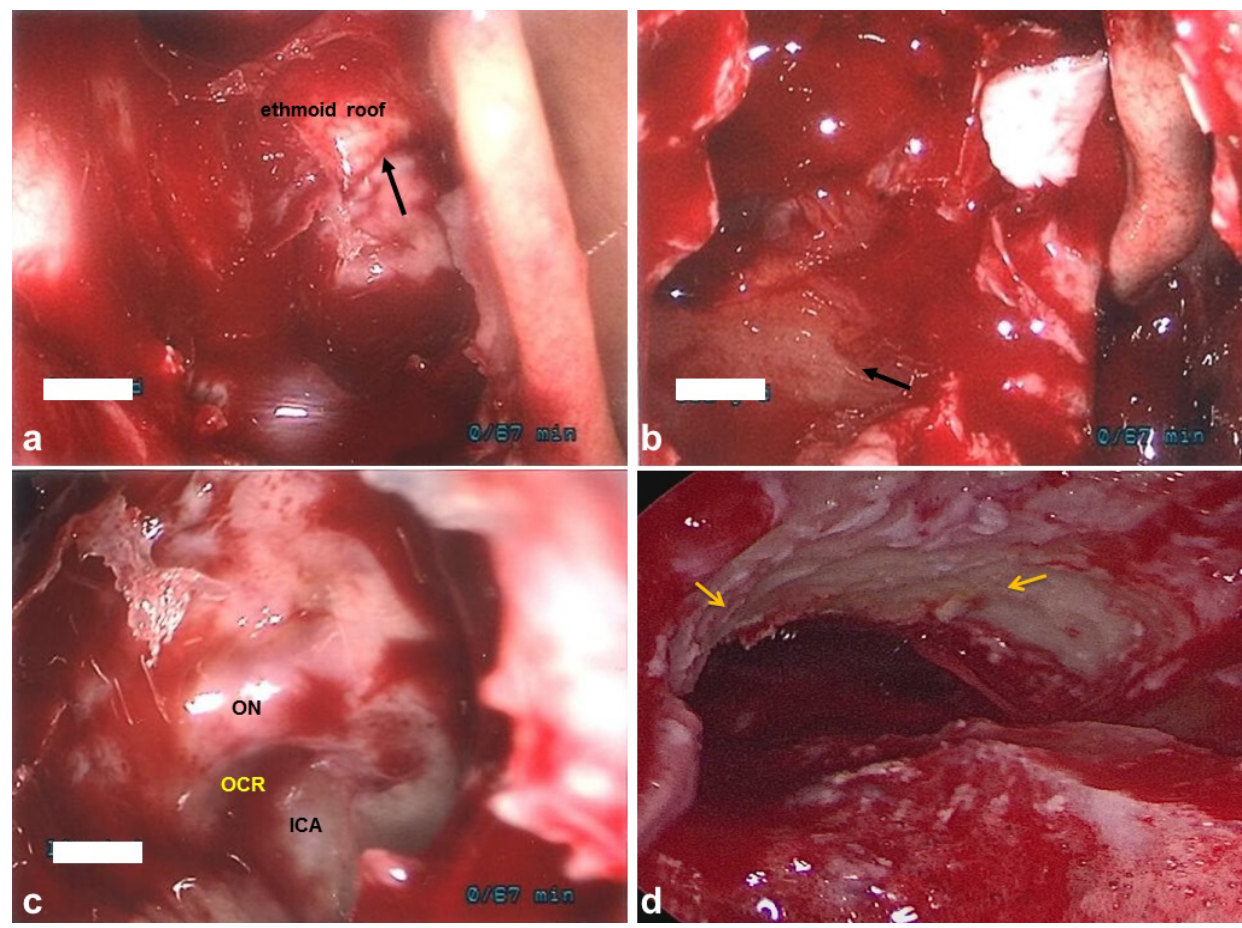

Figure 3. (a) superior boundary of resection is ethmoid roof (arrow:anterior ethmoid artery ); ( b ) the whole maxillary medial wall is removed ( black arrow: posterior antral wall ); ( c ) sphenoid sinus is dissected ( ON: optic nerve; ICA: internal carotid artery; OCR: optic carotid recess ); ( d ) Dissecting frontal sinus beyond frontal recess by outside-in Draf 3 approach (yellow arrow: periosteaum of frontal process of maxilla). 


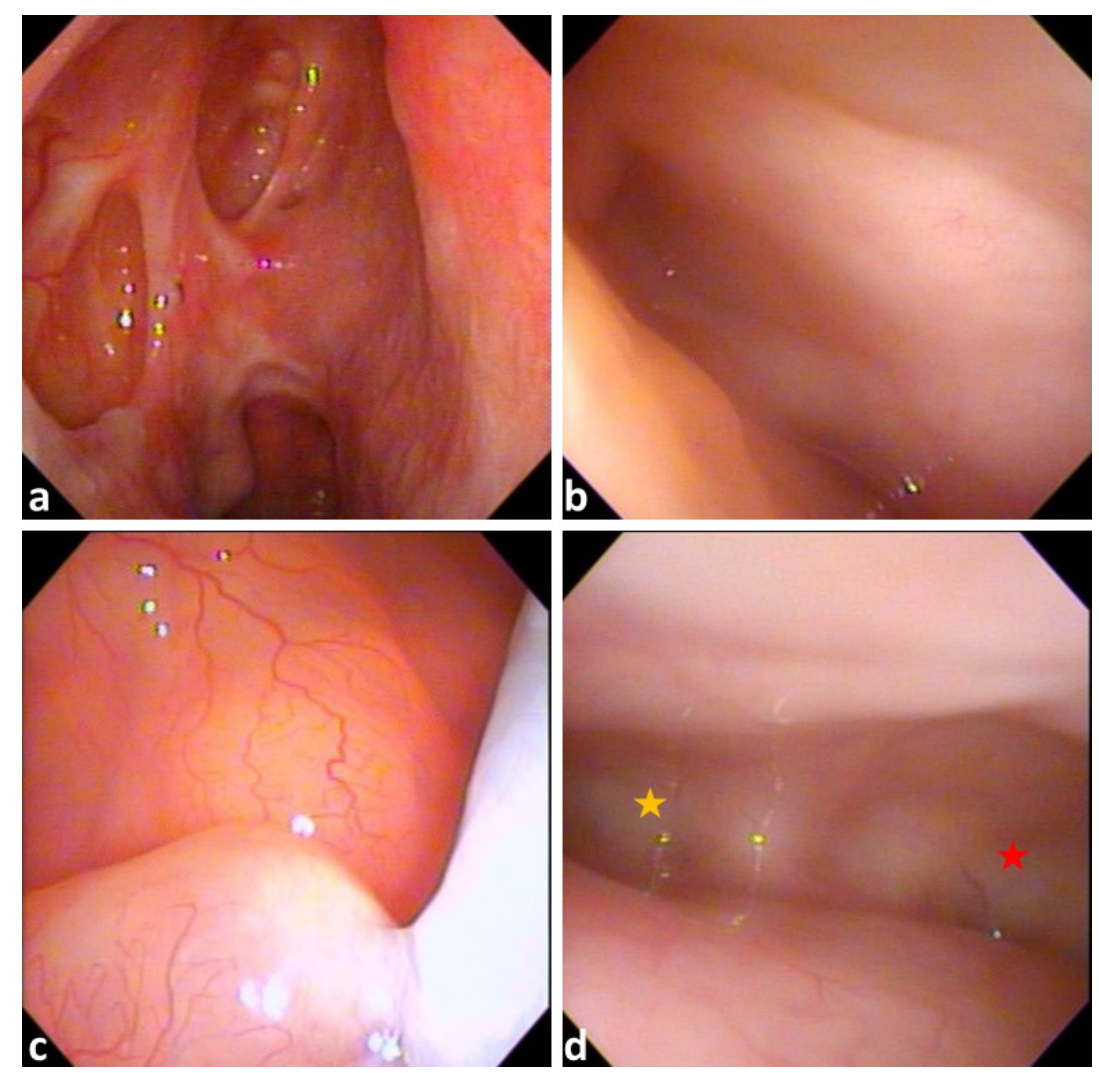

Figure 4. (a)There is no recurrent tumor 22 months after treatment. (b) right frontal sinus is disease free. (c) antrum mucosa is healthy. (d) There is no diseased mucosa in the frontal draning system (yellow asterisk: right frontal sinus; red asterisk: left frontal sinus).

\section{Conclusion}

Outside-in Draft 3 technique provides directly access to the frontal sinuses, particularly in situations lacking landmarks of frontal recess due to scarring or tumor infiltration. As comparing to conventional Draft 3 procedure, it could decrease the dependence for navigation system because the anterior limit of anterior skull base can be identified soonly; bony working is confined to a safer region. So, we believe this technique is quite reliable in combing with other procedures to manage majority of sino-nasasal tumors if navigation system is unavailable.

\section{Reference}

1. Knisely A, Barham HP, Harvey RJ, Sacks R (2015) Outside-In Frontal Drill-Out: How I Do It. Am J Rhinol Allergy 29: 397-400. [Crossref]

Copyright: $\odot 2017$ Wang T. This is an open-access article distributed under the terms of the Creative Commons Attribution License, which permits unrestricted use, distribution, and reproduction in any medium, provided the original author and source are credited. 